\title{
Dynamics of dislocations and surface instabilities in misfitting heteroepitaxial films
}

\author{
Mikko Haataja, ${ }^{1}$ Judith Müller, ${ }^{2}$ A. D. Rutenberg, ${ }^{3}$ and Martin Grant ${ }^{1}$ \\ ${ }^{1}$ Centre for the Physics of Materials, Department of Physics, McGill University, Rutherford Building, 3600 rue University, Montréal, \\ Québec H3A 2T8, Canada \\ ${ }^{2}$ Instituut-Lorentz for Theoretical Physics, University of Leiden, P.O. Box 9506, NL-2300 RA Leiden, The Netherlands \\ ${ }^{3}$ Department of Physics, Dalhousie University, Halifax, Nova Scotia B3H 3J5, Canada
}

(Received 6 June 2001; published 13 December 2001)

\begin{abstract}
We introduce a continuum model of elasticity in a nonequilibrium multiphase system-including smooth and singular strains, as well as their coupling to free surfaces-and apply it to the dynamics of misfitting heteroepitaxial films. Above a critical thickness, defects relieve strain, competing with an instability at the interface. Depending on their mobility, defects can screen stress by building up at large-curvature groove tips, leading to high ductility, or be "outrun" by the tips, leading to brittleness. Hence we find a nonequilibrium brittle to ductile transition.
\end{abstract}

DOI: 10.1103/PhysRevB.65.035401

PACS number(s): 62.20.Fe, 64.60.My, 68.55.-a

The properties of thin films can be tuned by growing them on a substrate that induces strain. However, such misfitting heteroepitaxial thin films can only be grown to a critical thickness. At that point, strain relaxes from the film as it either becomes plastic, or "buckles," and complex structure forms. Interest in this dynamical process goes back to the classic work of Frank and van der Merwe. ${ }^{1}$ If one considers small strains where buckling is unimportant, Matthews and Blakeslee $^{2}$ derived the critical thickness at which coherency is lost through the appearance of misfit dislocations, which accommodate the applied strain. Alternatively, for large strains relief can occur without plasticity as the free surface becomes corrugated ("buckles") coherently, as identified in the stability analyses of Asaro and Tiller, ${ }^{3}$ and of Grinfeld. ${ }^{4}$ This latter mechanism is associated with dislocation-free Stranski-Krastanow growth in subcritical films. ${ }^{5-11}$

In general both mechanisms are present. Misfit dislocations partially accommodate strain, and buckling occurs due to the residual strain. For example, controlled annealing experiments of Ozkan et al. ${ }^{12}$ on supercritical SiGe films have demonstrated that groove alignment from the buckling depends on the presence of misfit dislocations. It is theoretically challenging to address both plasticity and buckling in this nonequilibrium process; the former involves singular contributions to the strain, while the latter constitutes a freeboundary-value problem. A molecular dynamics study of Dong et al. ${ }^{13}$ addressed both aspects of the process and found a qualitative coupling between buckling and dislocation nucleation; the relaxation of misfit strain by dislocations occurred inside grooves, which came about by the relaxation of strain by buckling. Schwarz ${ }^{14}$ studied misfit dislocation lines in three-dimensional static films in a quasiequilibrium framework. Unfortunately, neither approach is viable for a quantitative study of the dynamics: molecular dynamics considers too short a time scale, and the quasiequilibrium approximation cannot explore how film geometry and dislocations evolve together. An alternate and appealing method is continuum mechanics, where the quantities entering constitutive relations, such as shear and bulk moduli, $\mu$ and $\kappa$, are straightforward to determine experimentally. Another benefit of such a coarse-grained approach is that the time evolution is on experimentally relevant scales.

Herein we introduce a continuum model of elasticity in a nonequilibrium multiphase system, including smooth and singular strains, as well as their coupling to free surfaces. To show the usefulness of this approach, we have studied thinfilm morphology from the initial stages of instability of supercritical films to the fully nonlinear late-time regime. We find that the initial instability persists even with dislocations, with a growth rate that depends on the defect core energy and mobility. In the late-time regime, an island morphology forms with deep grooves if the defects cannot accommodate all strain-this is similar to that seen for subcritical films. ${ }^{7,9,10}$ In contrast to the case of coherent buckling of thin films, we find that the dynamics of grooves are like those of crack tips in metals. Dislocations build up ahead of the groove tip, slowing it by screening some of the local stress. Furthermore, depending on the mobility of the dislocations, the film is either ductile (where the mobility is high enough that the defects keep pace with the growing groove tip) or brittle (where the groove tip outruns the dislocations). We find evidence for a dynamic brittle to ductile transition in groove dynamics, analogous to what occurs for cracks in fracture mechanics. ${ }^{15}$

Following Onuki and Nishimore, ${ }^{16}$ and two of us, ${ }^{9}$ we introduce a Ginzburg-Landau free energy functional in terms of the local phase and elastic interactions. The order parameter $\phi(\vec{r}, t)$, as a function of space $\vec{r}$ and time $t$, determines the equilibrium phase, vapor $\left(\phi_{\mathrm{eq}}=0\right)$, film $\left(\phi_{\mathrm{eq}}=1\right),{ }^{17}$ or substrate $\left(\phi_{\text {eq }}=2\right)$. Elasticity involves the singular and nonsingular parts of the strain tensor $\mathbf{u}$, or equivalently for the singular part, the dislocation's Burger's vector $\vec{b}$. The free energy is

$$
\mathcal{F}=\int_{\vec{r}}\left[\frac{\epsilon^{2}}{2}|\nabla \phi|^{2}+f(\phi)+f_{\text {elastic }}(\phi, \mathbf{u})\right],
$$

where the first term gives rise to a surface energy and welldefined interfaces on the length scale of $\epsilon$. The free energy is a functional of two fields; the phase field $\phi$ and the continu- 
ous Burger's vector $\vec{b}$. The singular stress is related to the Airy stress function $\chi[\vec{b}]$. The singular stress and the singular strain are related to each other by the usual elasticity relation. The three phases are described by $f(\phi)=a \phi^{4}(\phi$ $-1)^{2}(\phi-2)^{2}+\left(\eta^{2} / 2 \kappa\right) \Phi_{\text {misfit }}^{2}$, where a controls the surface-free energies. The externally applied misfit stress is proportional to ${ }^{9} \eta$, and $\Phi_{\text {misfit }}$ and $\Phi_{\text {solid }}$ are simple functions of $\phi$ (Ref. 18) required to ensure that misfit stresses exist only in the film and that the shear modulus $\mu \Phi_{\text {solid }}$ vanishes in the vapor phase. For simplicity, we choose identical elastic moduli for the two solid phases.

The elastic part of the free energy has several parts: $f_{\text {elastic }}=f_{\text {smooth }}+f_{\text {sing }}+f_{\text {core }}$. The smooth part of the elastic energy ${ }^{19,20}$ is defined in terms of the elastic moduli and the smooth strain fields $u_{i j}, f_{\text {smooth }}=\eta \Phi_{\text {misfit }} u_{k k}+\frac{1}{2} \kappa\left(u_{k k}\right)^{2}$ $+\mu \Phi_{\text {solid }}\left[u_{i j}-\left(\delta_{i j} / 2\right) u_{k k}\right]^{2}$, where a summation convention is implicit over repeated indices. The singular part of the elastic energy is $f_{\text {sing }}=\frac{1}{2} \kappa\left(u_{k k}^{s}\right)^{2}+\mu \Phi_{\text {solid }}\left[u_{i j}^{s}-\left(\delta_{i j} / 2\right) u_{k k}^{s}\right]^{2}$ $+u_{i j} \sigma_{i j}^{s}$, where the last term gives the coupling between smooth strain and singular stress $\sigma_{i j}^{s}$ defined below in terms of the Burger's vector $\vec{b}$. Depending on signs, the singular strain $u_{i j}^{s}$ is able to match and relieve the smooth strain. In the bulk phases where $\mu \Phi_{\text {solid }}$ and $\kappa$ are constants, stresses are proportional to strains, as given by Hooke's law.

Since time scales of interest are much longer than elastic relaxation times, we employ the local mechanical equilibrium conditions $\nabla_{j} \sigma_{i j}=0$. This permits us to integrate out the $\mathbf{u}$ to first order in $\mu / \kappa,{ }^{9,16}$ giving rise to long-range elastic forces. This gives $\sigma_{i j}^{s}=\epsilon_{i k} \epsilon_{j l} \nabla_{k} \nabla_{l} \chi$, where $\epsilon_{i j}$ denotes the antisymmetric unit tensor with $\epsilon_{x y}=1$, and $\chi$ is the Airy stress function, ${ }^{19,20}$ that gives rise to the Peach-Koehler force between dislocations. ${ }^{21}$ We restrict ourselves to two dimensions where $\chi$ is a scalar determined by a biharmonic equation in the different phases. ${ }^{22,23}$ Finally, the core energy of the defects is given by the sixth-order polynomial $f_{\text {core }}$ $=\frac{1}{2} E_{c}|\vec{b}|^{2}+c|\vec{b}|^{2}\left(b_{0}^{2}-|\vec{b}|^{2}\right)^{2}$, where we will call $E_{c}$ the energy of a dislocation, $c$ is a constant, and the dislocation strength is $b_{0}$.

The dynamics of $\phi$ and $\vec{b}$ are driven by minimization of $\mathcal{F}$. For the order parameter

$$
\begin{aligned}
\tau \frac{\partial \phi}{\partial t}= & -\frac{\delta \mathcal{F}}{\delta \phi}=\epsilon^{2} \nabla^{2} \phi-f^{\prime}(\phi)-\frac{\chi}{4} \Phi_{\text {solid }}^{\prime} \epsilon_{i j} \nabla_{i} b_{j} \\
& -\frac{16 \mu \eta^{2}}{\kappa^{2}}\left[2 \Phi_{\text {misfit }}^{\prime} m(\vec{r})+\Phi_{\text {solid }}^{\prime} n(\vec{r})\right] \\
& -\frac{\Phi_{\text {solid }}^{\prime} \chi^{2}}{2 Y l^{4}}-\beta(\vec{r})\left(\frac{Y}{4} \Phi_{\text {solid }}^{\prime} \epsilon_{i j} \nabla_{i} b_{j}-\frac{\Phi_{\text {solid }}^{\prime}}{l^{4}}\right),
\end{aligned}
$$

where primes on functions denote derivatives with respect to $\phi, \tau^{-1}$ is a mobility, $Y=4 \kappa \mu /(\kappa+\mu)$ is Young's modulus, $l$ is a microscopic length, ${ }^{23}$ and long-range elastic forces enter through $^{24} m(\vec{r})$ and $n(\vec{r})$. The coupling $\beta(\vec{r})$ $\equiv \epsilon_{i j} \delta F / \delta\left(\nabla_{i} b_{j}\right)$ between the dislocations and $\sigma_{i j}$ results

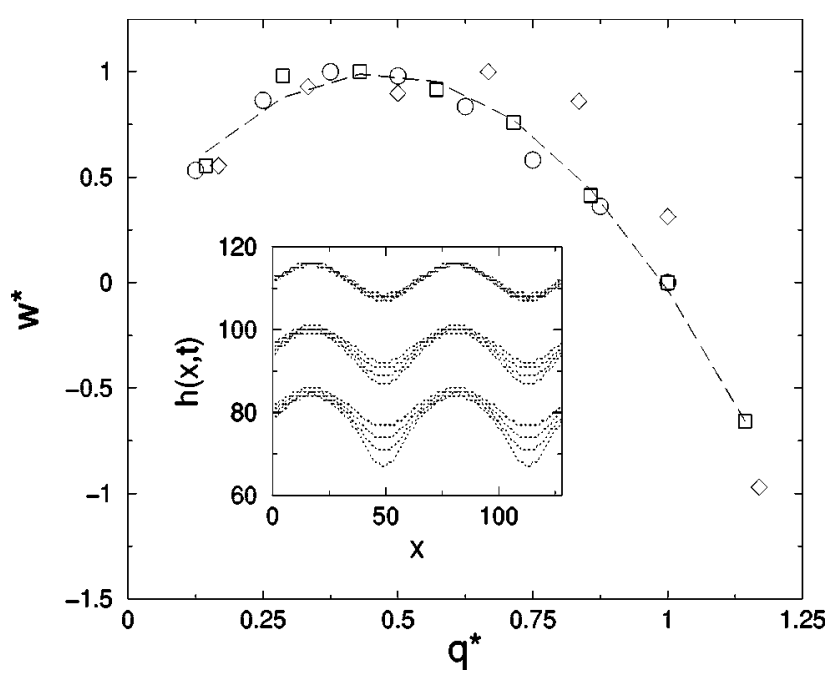

FIG. 1. Scaled amplitude of growth modes $\omega^{*}$ as a function of scaled wave number $q^{*}$ in the early-time linearly unstable regime. Diamonds $E_{c}=1$, squares $E_{c}=50$, circles $E_{c}=\infty$ (no defects), dashed line is linear stability theory. Data scaled by maximum growth rate and wave number for restabilization, both of which increase with increasing $E_{c}$. Inset shows configurations for $E_{c}$ $=1,50, \infty$, from top to bottom. Four times are shown for each; grooves deepen with time for all.

from integrating the strain out of the dynamics. ${ }^{22,23}$ For the dislocation densities

$$
\begin{aligned}
\frac{\partial b_{x}}{\partial t}= & \left(m_{g} \nabla_{x}^{2}+m_{c} \nabla_{y}^{2}\right) \frac{\delta \mathcal{F}}{\delta b_{x}} \\
= & \left(m_{g} \nabla_{x}^{2}+m_{c} \nabla_{y}^{2}\right)\left\{\frac{d f_{\text {core }}}{d b_{x}}+\frac{\epsilon_{x j}}{4} \nabla_{j}\right. \\
& \left.\times\left[(\chi+Y \beta) \Phi_{\text {solid }}\right]\right\},
\end{aligned}
$$

where $m_{g}$ and $m_{c}$ denote the glide and climb mobilities (for $b_{y}, m_{g}$ and $m_{c}$ are interchanged). We equilibrate the fields $\chi$ and $\beta$ with a mixed real space-spectral method ${ }^{23}$ every 10-30 $\Delta t$ time steps, and relax them dynamically after each time step with 50 Euler iterations. The phase field $\phi$ and dislocation densities $\vec{b}$ are updated using finite differencing on a scale of $\Delta x$ for the spatial gradients and the Euler method for time evolution. System sizes considered were $128 \times 128,256 \times 256$, and $64 \times 512$. For the results reported $\left(\Delta x, \Delta t, \epsilon, \kappa, l, \tau, m_{g}\right)=(1, \leqslant 0.01,1$,

$\left.1,2,1, m_{c}\right)$. The climb mobility $m_{c}$ was varied between 0.01 and 0.2 , the core energy was controlled by varying $E_{c}$ between 1 and 50, and dislocation densities were initialized with small random fluctuations of magnitude $10^{-4}$. We report results for the supercritical regime where nucleation of defects does not control evolution. The remaining parameters $\vec{P} \equiv\left(a, \eta, \mu, c, b_{0}\right)$ are reported in context below.

With our approach, the physics of the film under external stress is clear. The smooth strain fields adjust to the stress by buckling, while the singular part of the internal strain adjusts to the stress by nucleating dislocations, if the core energy of 


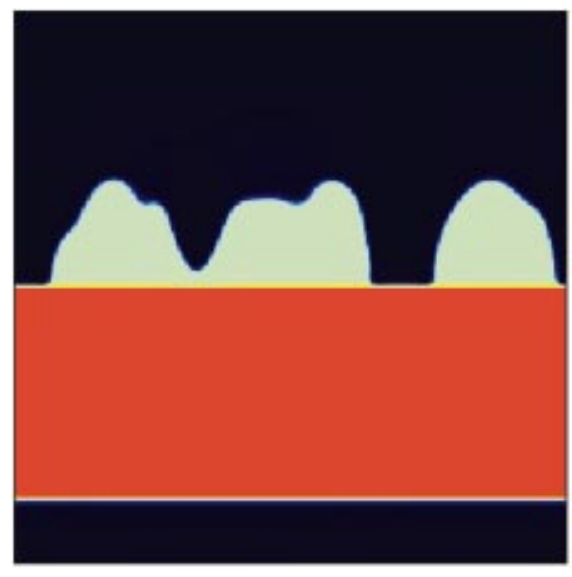

(a)

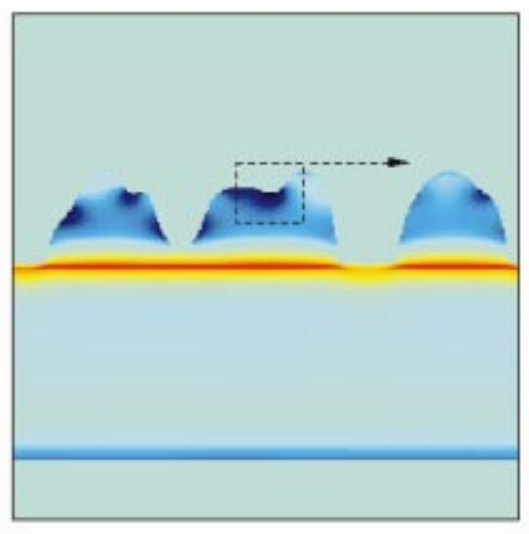

(c)

those defects is not too large. Whether or not the dislocations can move fast enough to track the accumulation of local stress at the moving boundary is dependent upon their mobilities. For very early times, linear stability analysis describes the evolution. In Fig. 1 we show the amplitude of the initial growth rate, for increasing core energies $E_{c}=1,50$, and $\infty, m_{c}=0.01$, and in the inset interface profiles at $t$ $\leqslant 25$. We initialized the system with a sinusoidal interface deformation of small amplitude $h_{0}$ and wave number $q$, with parameters $\vec{P}=\left(2,0.5,0.5,2 \times 10^{6}, 0.01\right)$. At early times, we observe the linear stability regime where $h(q, t) \propto \exp \omega(q) t$, with $\omega=e^{2} q-\gamma q^{2}$, where $e$ and $\gamma$ are proportional to the external strain and film-vapor surface tension, respectively. Defects shorten the period over which this regime can be seen, and renormalize $e$ and $\gamma .{ }^{25}$

For later times, coarsening occurs at the film-vapor interface, leading to a grooved profile as shown in Fig. 2(a) (with defects $E_{c}=10$ ) and Fig. 2(b) (without defects $E_{c}=\infty$ ) at time $t=80$, and $m_{c}=0.0125$. Parameters are $\vec{P}$ $=\left(1,0.775,0.25,2 \times 10^{5}, 0.1\right)$. Eventually, the grooves cause the film to break up into islands leaving the substrate par- tially exposed. Indeed, this is what is observed in the controlled annealing experiments of Ozkan et al. ${ }^{12}$ Examination of the $b_{x}$ field shown in Fig. 2(c) reveals considerable dislocation activity at the film-substrate interface (thick red line). In particular, dislocations with $b_{x}>0$ align themselves at the film-substrate interface to partially screen the film tension. In the island regime, a large dislocation density accumulates at the bottom of each island, exactly as seen in the experiments. ${ }^{12}$

A striking feature of these late-time configurations is the defect concentration at the bottom of the grooves [Fig. 2(d)]. These dislocations partially relax the stress concentration and, therefore, slow growth. To address this in more detail, we simulated narrow systems with a single groove, $E_{c}=1$, and varying $m_{c}$. See Fig. 3: top of figure with parameters $\vec{P}=\left(1,0.775,0.25,2 \times 10^{3}, 0.1\right)$ for $m_{c}=0.05$ and times $75-$ 100 ; bottom of figure with parameters $\vec{P}=(1,0.9,0.25,1$ $\times 10^{5}, 0.04$ ) for $m_{c}=0.2$ and times $12-24$ (little evolution occurs after this time). We initially notched the system and then tracked its growth under stress. The groove attained a well-defined steady-state velocity and shape. Dislocation distributions are clearly evident. 

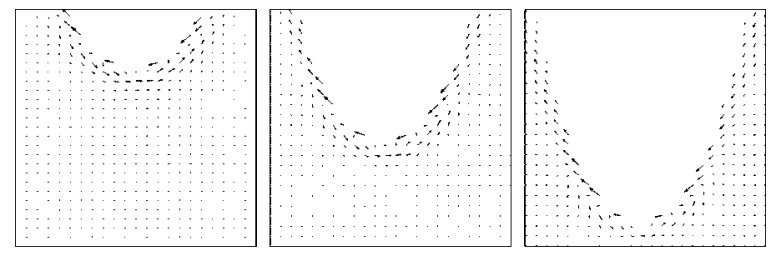

(a)
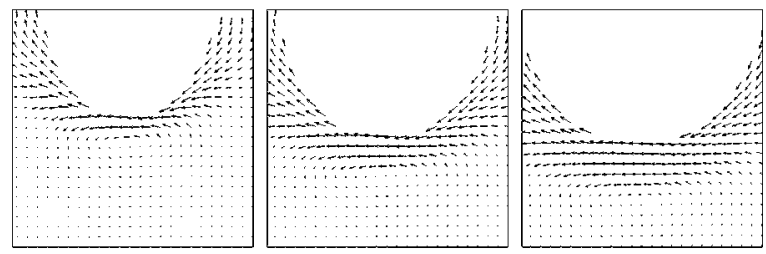

(b)

FIG. 3. (a) Sequence of groove tips in brittle region, outrunning dislocations, which are shown as Burger's vectors. (b) Sequence of groove tips in ductile region, where dislocations keep pace with tip.

Two main effects are taking place. First, the high concentration of stress at the groove tip causes defects to nucleate and relax some of the stress, ${ }^{26}$ if the core energy $E_{c}$ is not too high. This slows the groove, and blunts its tip. Second, depending on the mobility of the dislocations, the groove tip can "outrun" the defects. Low dislocation mobility leads to a fast tip velocity, where the defects lag behind the tip, while a high mobility gives rise to a slow tip velocity, since the stress-relieving defects can keep pace with the tip. In other words, if the mobility of the dislocations is too low, the film is brittle, while it is ductile for high mobilities. See Fig. 4. For small $E_{c}$ (defect nucleation is facilitated), we observe two distinct groove-tip speeds ${ }^{25} v_{\min }$ and $v_{\max }$, for $m_{c} \gg 1$ and $m_{c} \ll 1$, respectively. Defining a temperature through an Arrhenius form $m_{c} \sim \exp \left(-E_{b} / k_{B} T\right)$, gives the temperature for a brittle-ductile transition: for $T \gg T_{\mathrm{BDT}} \sim E_{b} / k_{B}, v_{\text {tip }}$

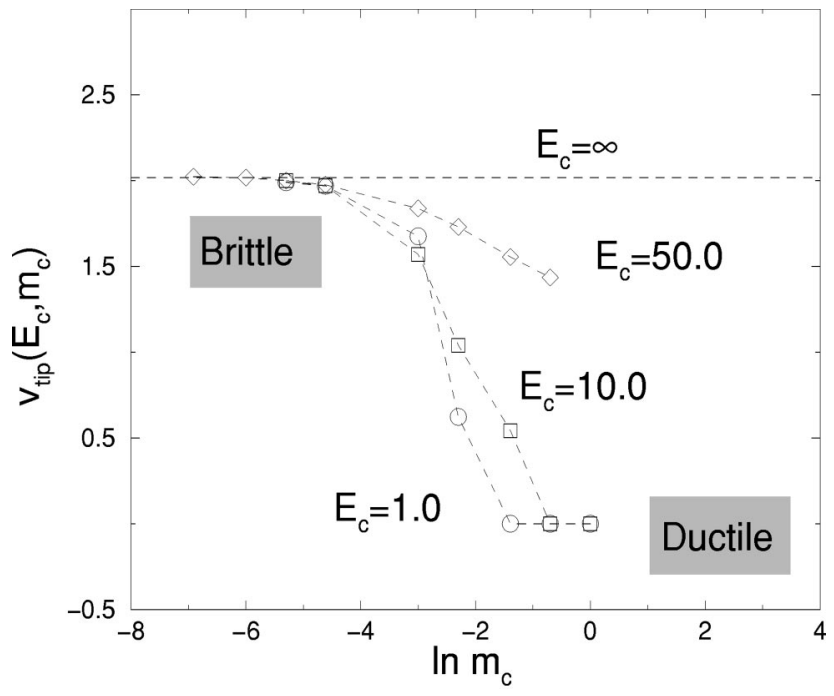

FIG. 4. Tip speed vs natural log of climb mobility for various $E_{c}$ as indicated. Note well-defined transition from brittle region to ductile region with increasing mobility.

$\approx v_{\text {min }}$, whereas for $T \ll T_{\mathrm{BDT}}, v_{\text {tip }} \approx v_{\text {max }}$. This scenario is analogous to what is observed in materials undergoing fracture; for example, Gumbsch et al. ${ }^{15}$ studied fracture in tungsten single crystals, and concluded that the brittle-ductile transition required sufficiently mobile dislocations.

To conclude, we have introduced a continuum model of elasticity in a multiphase system, which includes both smooth and singular strains, as well as the coupling of those strains to free surfaces. In the future, we shall extend this formalism to consider a tensorial Airy stress function, with three independent components, as is required for three dimensions.

This work was supported by the Natural Sciences and Engineering Research Council of Canada, le Fonds pour la Formation de Chercheurs et l'Aide à la Recherche du Québec, and, in part, by the Academy of Finland ( $\mathrm{MH})$. We thank Mohsen Sabouri-Ghomi and Martin Dubé for useful discussions.
${ }^{1}$ F.C. Frank and J.H. van der Merwe, Proc. R. Soc. London, Ser. A 198, 205 (1949).

${ }^{2}$ J.W. Matthews and A.E. Blakeslee, J. Cryst. Growth 27, 118 (1974).

${ }^{3}$ R.J. Asaro and W.A. Tiller, Metall. Trans. 3, 1789 (1972).

${ }^{4}$ M. Grinfeld, Dokl. Akad. Nauk SSSR 265, 836 (1982); Europhys. Lett. 22, 723 (1993), and references therein.

${ }^{5}$ D.J. Srolovitz, Acta Metall. 37, 621 (1989).

${ }^{6}$ B.J. Spencer, P.W. Voorhees, and S.H. Davis, Phys. Rev. Lett. 67, 3696 (1991).

${ }^{7}$ W.H. Yang and D.J. Srolovitz, Phys. Rev. Lett. 71, 1593 (1993).

${ }^{8}$ J.E. Guyer and P.W. Voorhees, Phys. Rev. Lett. 74, 4031 (1995).

${ }^{9}$ J. Müller and M. Grant, Phys. Rev. Lett. 82, 1736 (1999).

${ }^{10}$ K. Kassner and C. Misbah, Europhys. Lett. 46, 217 (1999); K. Kassner, C. Misbah, J. Müller, J. Kappey, and P. Kohlert, Phys.
Rev. E 63, 036117 (2001)

${ }^{11}$ H. Gao and W.D. Nix, Annu. Rev. Mater. Sci. 29, 173 (1999).

${ }^{12}$ C.S. Ozkan, W.D. Nix, and H. Gao, Appl. Phys. Lett. 70, 2247 (1997).

${ }^{13}$ L. Dong, J. Schnitker, R.W. Smith, and D.J. Srolovitz, J. Appl. Phys. 83, 217 (1998).

${ }^{14}$ K.W. Schwarz, J. Appl. Phys. 85, 108 (1999); 85, 120 (1999).

${ }^{15}$ P. Gumbsch, J. Riedle, A. Hartmeier, and H.F. Fischmeister, Science 282, 1293 (1998).

${ }^{16}$ A. Onuki and H. Nishimori, Phys. Rev. B 43, 13649 (1991); see also, C. Sagui, A.M. Somoza, and R.C. Desai, Phys. Rev. E 50, 4865 (1994).

${ }^{17}$ We do not consider facets, which can lead to misfit nucleation without roughening: J. Tersoff and F.K. LeGoues, Phys. Rev. Lett. 72, 3570 (1994). 
${ }^{18}$ Generalizing the approach of R. Kobayashi, Physica D 63, 410 (1993), the $\Phi$ 's enforce convenient thermodynamics for the vapor, film, and substrate equilibrium phases $\left(\phi_{\mathrm{eq}}=0,1\right.$, or 2 , respectively). Namely, we require $\Phi^{\prime}\left(\phi_{\text {eq }}\right)=0$, where the prime denotes a derivative, and $\Phi\left(\phi_{\text {eq }}\right)=\phi_{\text {eq }}$, for $\phi_{\text {eq }}=0$ or 1 , while for $\phi_{\text {eq }}=2, \Phi_{\text {misfit }}=0$ and $\Phi_{\text {solid }}=1$. These are satisfied by the choices $\Phi_{\text {misfit }}=-\frac{1}{11}\left(2 \phi^{6}-12 \phi^{5}+15 \phi^{4}+20 \phi^{3}-36 \phi^{2}\right)$, and $\Phi_{\text {solid }}=-\frac{3}{132}\left(19 \phi^{6}-81 \phi^{5}+60 \phi^{4}+135 \phi^{3}-177 \phi^{2}\right)$.

${ }^{19}$ L. Landau and E.M. Lifshitz, Theory of Elasticity, 2nd ed. (Pergamon Press, Oxford, 1986).

${ }^{20}$ F.R.N. Nabarro, Theory of Crystal Dislocations (Dover Publications, New York, 1967).

${ }^{21}$ D.R. Nelson and B.I. Halperin, Phys. Rev. B 19, 2457 (1979).

${ }^{22}$ M. Haataja, Ph.D. thesis, McGill University, 2001; M. Haataja, J. Müller, A. D. Rutenberg, and M. Grant (unpublished).

${ }^{23}$ The Airy stress function $\chi$ is found from $\nabla^{4} \chi+\left(1 / l^{4}\right)(1$ $\left.-\Phi_{\text {solid }}\right) \chi=(Y) \Phi_{\text {solid }} \epsilon_{i j} \nabla_{i} b_{j}$, which incorporates the necessary boundary conditions and thickness-dependent dislocation selfenergy, where $Y$ denotes the Young's modulus, and $l$ is a small length of order the interface thickness. This ensures, for ex- ample, that the defects have only short-range interactions $\sim l$ in the vapor phase (defect reservoir), and that they interact via the Peach-Koehler force in the solid phases. Also, note that the back-coupling variable $\beta(\vec{r}) \equiv \epsilon_{i j} \delta F / \delta\left(\nabla_{i} b_{j}\right)$ satisfies

$$
\begin{aligned}
\nabla^{4} \beta(\vec{r}) & +\left(1 / l^{4}\right)\left[1-\Phi_{\text {solid }}(\vec{r})\right] \beta(\vec{r}) \\
= & \left(\delta_{i j} / 4 \kappa\right) \nabla^{2} \sigma_{i j}(\vec{r})+(1 / 8 \mu) \\
& \times\left[\varepsilon_{i k} \varepsilon_{j l} \nabla_{k} \nabla_{l} \sigma_{i j}(\vec{r})-\left(\delta_{i j} / 2\right) \nabla^{2} \sigma_{i j}(\vec{r})\right] .
\end{aligned}
$$

${ }^{24}$ The smooth strains give rise to long-range forces via $m$ $\equiv \int_{\vec{r}^{\prime}, \vec{r}^{\prime \prime}} G\left(\vec{r}, \vec{r}^{\prime}\right) \nabla_{i}^{\prime} \nabla_{j}^{\prime}\left[\Phi_{\text {solid }}\left(\vec{r}^{\prime}\right) M_{i j}\left(\vec{r}^{\prime}, \vec{r}^{\prime \prime}\right) \Phi_{\text {misfit }}\left(\vec{r}^{\prime \prime}\right)\right]$, and, $n$ $\equiv\left[\Phi_{\text {misfit }}(\vec{r}) \delta_{i j} / 2-\nabla_{i} \nabla_{j} \int_{r^{\prime}} G\left(\vec{r}, \vec{r}^{\prime}\right) \Phi_{\text {misfit }}\left(\vec{r}^{\prime}\right)\right]^{2}$, where $\nabla^{2} G\left(\vec{r}, \vec{r}^{\prime}\right)=\delta\left(\vec{r}-\vec{r}^{\prime}\right) \quad$ and $\quad M_{i j}\left(\vec{r}^{\prime}, \vec{r}^{\prime \prime}\right)=\nabla_{i} \nabla_{j} G\left(\vec{r}^{\prime}, \vec{r}^{\prime \prime}\right)-\delta\left(\vec{r}^{\prime}\right.$ $\left.-\vec{r}^{\prime \prime}\right) \delta_{i j} / 2$.

${ }^{25}$ Of course, if the dislocations are sufficiently mobile no buckling instability takes place (Ref. 2).

${ }^{26}$ Defects are generated in pairs close to the interface, but the ones that increase the local strain diffuse into the vapor phase. 